\title{
天然富硒区广西壮族自治区桂平市水槄土壤 线虫群落结构特征及其指示意义
}

宋佳平 ${ }^{1}$, 袁林喜 $^{2}$, 刘晓东 ${ }^{1}$, 刘永贤 ${ }^{3}$, 王张民 ${ }^{4}$, 陈清清 ${ }^{4}$, 张泽洲 ${ }^{4}$, 龙泽东 ${ }^{5}$, 林锦铨 ${ }^{1}$, 尹雪斌, ${ }^{1,4 *}$

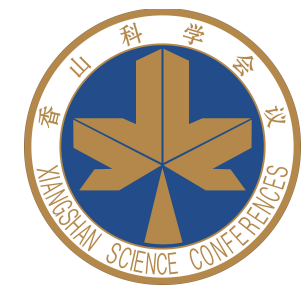

1. 中国科学技术大学地球和空间科学学院, 合肥 230026 ;

2. 西交利物浦大学健康与环境科学系, 苏州 215123 ;

3. 广西壮族自治区农业科学院农业资源与环境研究所, 南宁 530007;

4. 江苏省硒生物工程技术研究中心, 苏州 215123;

5. 湖南省农业科学院土壤肥料研究所, 长沙 410125

* 联系人, E-mail: xbyin@ustc.edu.cn

2021-03-05 收稿, 2021-04-04 修回, 2021-04-06 接受, 2021-04-07 网络版发表

广西创新驱动发展科技重大专项(桂科AA17202026)资助

摘要发展富硒农业对改善人体健康状况具有重要意义, 但目前尚未开展富硒土壤中生态安全评估相关研究. 土 壤生物是土壤物质循环和能量流动过程的主要调节者, 线虫是土壤中数量最丰富的后生动物, 其群落结构和多样 性对于土壤生态系统的能流通道和环境质量等均具有重要的指示意义. 本研究选取广西壮族自治区桂平市水稻土 壤(总硒为 100 900 $\mu \mathrm{g} / \mathrm{kg}$ ) 为研究对象, 将其分成低硒组、中硒组和高硒组, 对其中的线虫群落进行了分析. 结果显 示, 不同硒含量土壤中, 线虫群落结构差异显著; 相比于低硒组, 中硒组及高硒组线虫密度显著增大, $c p-2$ 线虫及食 真菌线虫相对丰度显著减小, F/B值和结构指数SI值显著减小; 随土壤总硒及有效硒含量的增加, 线虫密度、连胃 属(Chronogaster)线虫相对丰度、棱咽属(Prismatolainus)线虫相对丰度增大，拟丽突属(Acrobeloides)线虫相对丰 度、食真菌线虫相对丰度 $(\mathrm{Fu}) 、 \mathrm{~F} / \mathrm{B}$ 值和结构指数SI减小. 硒抑制了群落中的硒敏感线虫, 简化了土壤食物网结构. 在野外条件下线虫群落对于水稻土壤硒元素具有敏感的响应, 可作为水稻土壤硒素水平的生物指示者. 本研究为 科学评价天然富硒水稻土壤的生态安全提供了数据参考.

关键词水稻土壤, 硒, 线虫, 结构指数, 拟丽突属, 生物指示者

硒是人和动物必需的微量元素，人体硒摄人量过 低时会引起克山病、大骨节病等疾病 ${ }^{[1,2]}$. 中国卫生与 健康委员会推荐成人日硒摄人量为 $60 \mu \mathrm{g} / \mathrm{d}^{[3]}$, 而中国 $72 \%$ 地区的居民仍处于硒摄人量不足的状态 ${ }^{[4]}$. 为解决 这一问题，人们在天然富硒区利用土壤资源生产天然 富硒农产品 ${ }^{[5]}$, 在芬兰、新西兰、中国等缺硒地区施 用硒肥来提高作物中的硒含量, 从而改善缺硒人群的
硒摄人水平 ${ }^{[6-9]}$. 但是, 目前的研究仅关注提高农产品 中的硒含量, 对利用天然富硒土壤或施用硒肥是否对 土壤生态系统健康造成影响, 知之甚少. 为了保障天然 富硒土壤资源的可持续利用，我们在实际生产和研究 过程中找到能有效指示富硒土壤生态健康的生物指示 者是非常有必要的.

线虫是土壤生态系统中数量最多的后生动物, 直

引用格式: 宋佳平, 袁林喜, 刘晓东, 等. 天然富硒区广西壮族自治区桂平市水稻土壤线虫群落结构特征及其指示意义. 科学通报, 2022, 67: 537-547 Song J P, Yuan L X, Liu X D, et al. Nematode structure and its indication in natural selenium-rich paddy soil of Guiping City, Guangxi Zhuang Autonomous Region (in Chinese). Chin Sci Bull, 2022, 67: 537-547, doi: 10.1360/TB-2021-0118 
接参与到有机物质积累和营养物质循环过程. 随着人 们对土壤线虫的多样性及其生态重要性的广泛关注, 线虫越来越多地被用于土壤环境质量监控. 线虫各类 群敏感性存在明显差异, 可以对环境因子扰动作出不 同的响应，线虫密度、线虫属及各种群落生态指数(如 富集指数EI、通道指数NCR、成熟指数MI、结构指数 SI等)能很好地指示自然和农业土壤生态系统受干扰程 度 $^{[10 \sim 12]}$. 线虫对于重金属污染具有灵敏的响应 ${ }^{[13 ~ 16]}$, 如 在乌兹别克斯坦Almalyk工业园区进行的研究发现, 随 着土壤样品与污染源(主要污染物为 $\mathrm{As} 、 \mathrm{Cd} 、 \mathrm{Cu} 、 \mathrm{~Pb}$ 和 Zn)距离的减小, 土壤线虫的密度、生物量及成熟度 指数都明显下降 ${ }^{[17]}$. 番茄植株经含低浓度重金属(As、

$\mathrm{Cd} 、 \mathrm{Cu} 、 \mathrm{~Pb}$ )的污水灌溉18周后, 相较于对照组, 其对 应土壤中线虫的成熟度指数和结构指数均显著下 降 ${ }^{[16]}$. 目前有限的研究显示, 硒添加会对土壤线虫的数 量和群落结构产生影响. 如Bakonyi等人 ${ }^{[18]}$ 研究发现, 与对照组相比, 实验组 (醋酸铵EDTA提取态硒, $11 \mathrm{mg} / \mathrm{kg}$ ) 土壤线虫群落线虫属数、 $c p 3-5$ 类群线虫和 捕食性线虫数量显著降低, 这主要是由于高 $c-p$ 值线虫 和杂捕食线虫体型大、世代时间长，能快速对高浓度 硒扰动作出响应. 但目前尚缺乏探讨天然富硒土壤环 境中线虫对硒浓度变化响应的相关研究.

本研究对广西壮族自治区桂平市天然富硒区水稻 土壤线虫群落进行分析，比较不同硒含量下土壤线虫 群落结构差异, 分析土壤硒含量与多种线虫生态指标 的相关性，以期通过研究线虫对土壤硒浓度变化的响 应, 篮选对硒敏感的生物指标, 为利用线虫指标评价土 壤硒水平, 并进而评估天然富硒土壤生态健康提供科 学依据. 同时, 探究硒对线虫群落特征的影响, 加深对 土壤生物多样性和生态功能的全面认识.

\section{1 方法和材料}

\section{1 样品采集}

研究区域桂平市位于广西壮族自治区东南部, 属 南亚热带季风气候, 年平均气温为 $21.8^{\circ} \mathrm{C}$, 年平均降雨 量为 $1735 \mathrm{~mm}$, 土壤主要类型为湿润富铁土和湿润雉形 土. 2018 年5月(此时水稻处于分临期, 土壤水分较少, 便 于样品采集), 用土钻在研究区采集 $0 \sim 20 \mathrm{~cm}$ 的表层稻 田土壤样品(图1), 每个采样点随机采集3个土样, 混匀 作为一个样品放人无菌密封袋, 共采集样品 45 个, 并尽 快运回实验室均匀分成两份, 一份置于 $4^{\circ} \mathrm{C}$ 环境下保存
进行线虫分离及鉴定，一份置于实验室内自然风干以 备硒检测.

\section{2 土壤硒分析}

将自然风干的土壤样品研磨、去除石子和动植物 残骸并过 100 目篮子. 称取 $0.2 \mathrm{~g}$ 样品于雉形瓶中, 加人 $8 \mathrm{~mL}$ 浓硝酸和 $2 \mathrm{~mL}$ 高氯酸, 消化过夜. 第二天将雉形瓶 放在电热板上梯度加热至冒白烟, 取下冷却. 然后, 用 去离子水冲洗雉形瓶瓶壁后再次放在电热板二次加热. 冷却至室温, 加人 $5 \mathrm{~mL}$ 浓盐酸, 摇匀后放置反应过夜. 转移至 $25 \mathrm{~mL}$ 比色管内, 定容至刻度线, 利用原子苂光 分光光度计进行土壤总硒检测 ${ }^{[19]}$. 利用国家土壤标准 物质(GBW 07402-GSS2, $\mathrm{Se}=160 \pm 40 \mu \mathrm{g} / \mathrm{kg}$ )进行质 量控制, 此方法的回收率为 $91.5 \%$ 107.1\%. 土壤有效硒 分析参考Zhao等人 ${ }^{[20]}$ 的方法, 使用 $0.1 \mathrm{~mol} / \mathrm{L}$ 的磷酸二 氢钾作为提取剂提取后检测上清液硒含量.

\section{3 土壤线虫群落特征分析}

土壤线虫由南京农业大学土壤生态实验室进行鉴 定, 具体方法简述如下 ${ }^{[21,22]}$ : 新鲜土样剔除土壤根系后 采用蔗糖离心浮选法分离线虫, 用500目篮网收集线虫, 并用 $4 \%$ 的甲醛保存. 线虫在显微镜下计数, 每个样本随 机取 100 条线虫进行属鉴定, 少于 100 条线虫的样品, 对 全部线虫进行鉴定. 根据形态特征将线虫划分为植食 线虫 $(\mathrm{PF})$ 、食细菌线虫 $(\mathrm{Ba})$ 、食真菌线虫 $(\mathrm{Fu})$ 和杂/捕 食线虫 $(\mathrm{OP}) 4$ 个营养类群, 并根据生活史特征赋予范围 $1 \sim 5$ 的 $c-p$ 值 ${ }^{[23]}$.

采用多种生态指数对线虫多样性和群落结构进行 描述 ${ }^{[24,25]}$, 计算公式如下:

成熟度指数 $\mathrm{MI}=v_{i} \times f_{i}$,

通道指数 $\mathrm{NCR}=\mathrm{Ba} /(\mathrm{Ba}+\mathrm{Fu})$,

富信指数 $\mathrm{EI}=100 \times e /(e+b)$,

结构指数 $\mathrm{SI}=100 \times s /(s+b)$,

$\mathrm{F} / \mathrm{B}$ 指数 $\mathrm{F} / \mathrm{B}=\mathrm{Fu} / \mathrm{Ba}$,

多样性指数 $H^{\prime}=-\sum g_{i} \ln \left(g_{i}\right)$,

均匀度 $J^{\prime}=H^{\prime} / \ln (S)$,

营养多样性指数 $\mathrm{TD}=1 /\left(\sum P_{i}^{2}\right)$,

其中, $v_{i}$ 为第 $i$ 个线虫属线虫的 $c-p$ 值(除去植食性线虫), $f_{i}$ 是第 $i$ 个线虫属的相对丰度(除去植食性线虫); $e=\sum k_{e} n_{e}$, $s=\sum k_{s} n_{s}, b=\sum k_{b} n_{b}, k$ 代表被赋予给 $e\left(\mathrm{Ba}_{1}, \mathrm{Fu}_{2}\right), s\left(\mathrm{Ba}_{3}\right.$ 工 


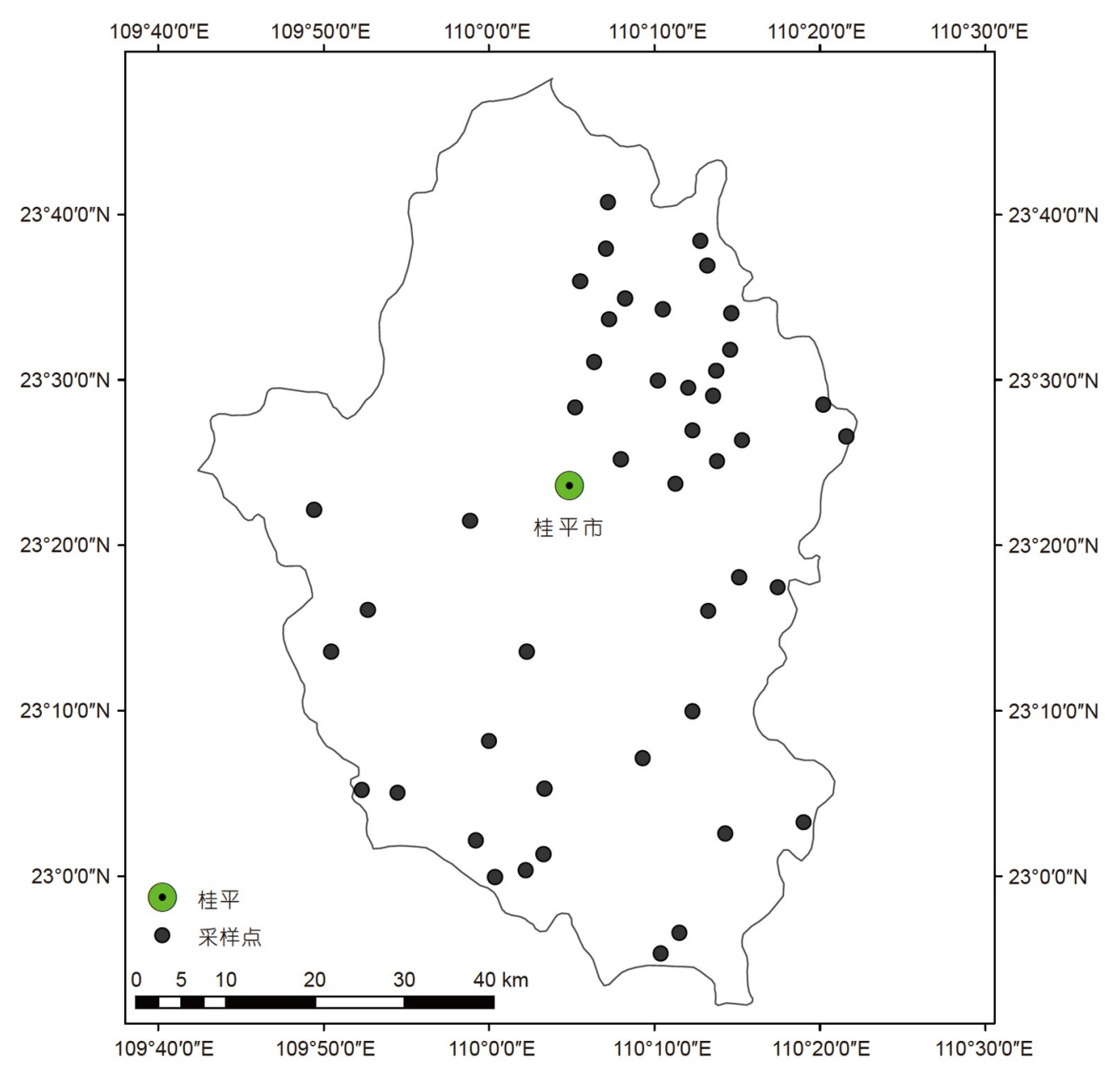

图 1 (网络版彩色)采样点分布图. 采样时间: 2018年5月

Figure 1 (Color online) Distribution of sampling sites. Sampling time: May 2018

$\left.\mathrm{Ba}_{5}, \mathrm{Fu}_{3} \sim \mathrm{Fu}_{5}, \mathrm{Om}_{3} \sim \mathrm{Om}_{5}, \mathrm{Pr}_{2} \sim \mathrm{Pr}_{5}\right)$ 以及 $b\left(\mathrm{Ba}_{2}, \mathrm{Fu}_{2}\right)$ 的权重 系数, $n$ 代表这些线虫的相对丰度; $g_{i}$ 是第 $i$ 个属线虫相对 丰度, $S$ 是线虫总属数, $P_{i}$ 是第 $i$ 个线虫营养群相对丰度.

\section{4 统计分析}

采用单因素方差分析法(one-way ANOVA)分析不 同硒含量土壤线虫密度、分类群组成及群落生态指数 差异, 采用Duncan法进行多重检验，显著性水平设 $\alpha=$ 0.05 , 使用SPSS 25.0进行数据统计分析, 利用Origin 2017软件完成作图. 基于不同线虫属丰度的Bray-Curtis 相似性矩阵, 用PRIMER 5.2.8软件通过非度量多维尺度 法(non-metric multidimensional scaling, NMDS)分析不同 硒含量土壤线虫群落结构差异. 使用R语言 corrplot包对 环境因子和线虫指标进行了皮尔森相关性分析并绘图.

\section{2 结果}

\section{1 土壤硒含量特征}

土壤总硒含量和有效硒含量见图2. 结果显示, 总硒 含量在100 900 $\mu \mathrm{g} / \mathrm{kg}$ 范围内, 平均值为 $360 \mu \mathrm{g} / \mathrm{kg}$, 接近 世界土壤平均硒含量 $(400 \mu \mathrm{g} / \mathrm{kg})$. 根据李家熙和张光 弟 ${ }^{[26]}$ 对全国土壤地球化学的调查数据, 土壤被划分为极 低硒土壤( $<100 \mu \mathrm{g} / \mathrm{kg})$ 、低硒土壤(100 200 $\mu \mathrm{g} / \mathrm{kg})$ 、中 硒土壤 $(200 \sim 400 \mu \mathrm{g} / \mathrm{kg})$ 、高硒土壤 $(>400 \mu \mathrm{g} / \mathrm{kg})$. 本研究 土壤样品据此可分为低硒组 $(n=8$, 占比 $17.8 \%)$ 、中硒 组 $(n=23$, 占比 $51.1 \%)$ 和高硒组 $(n=14$, 占比 $31.1 \%)$. 土 壤有效硒含量在 $1.2 \sim 43.9 \mu \mathrm{g} / \mathrm{kg}$ 范围内, 平均值为 $17.4 \mu \mathrm{g} / \mathrm{kg}$, 有效硒占比为 $0.6 \% \sim 15.0 \%$, 平均值为 $5.1 \%$. 随总硒含量增加, 有效硒含量也随之增加 $(P<0.05)$. 

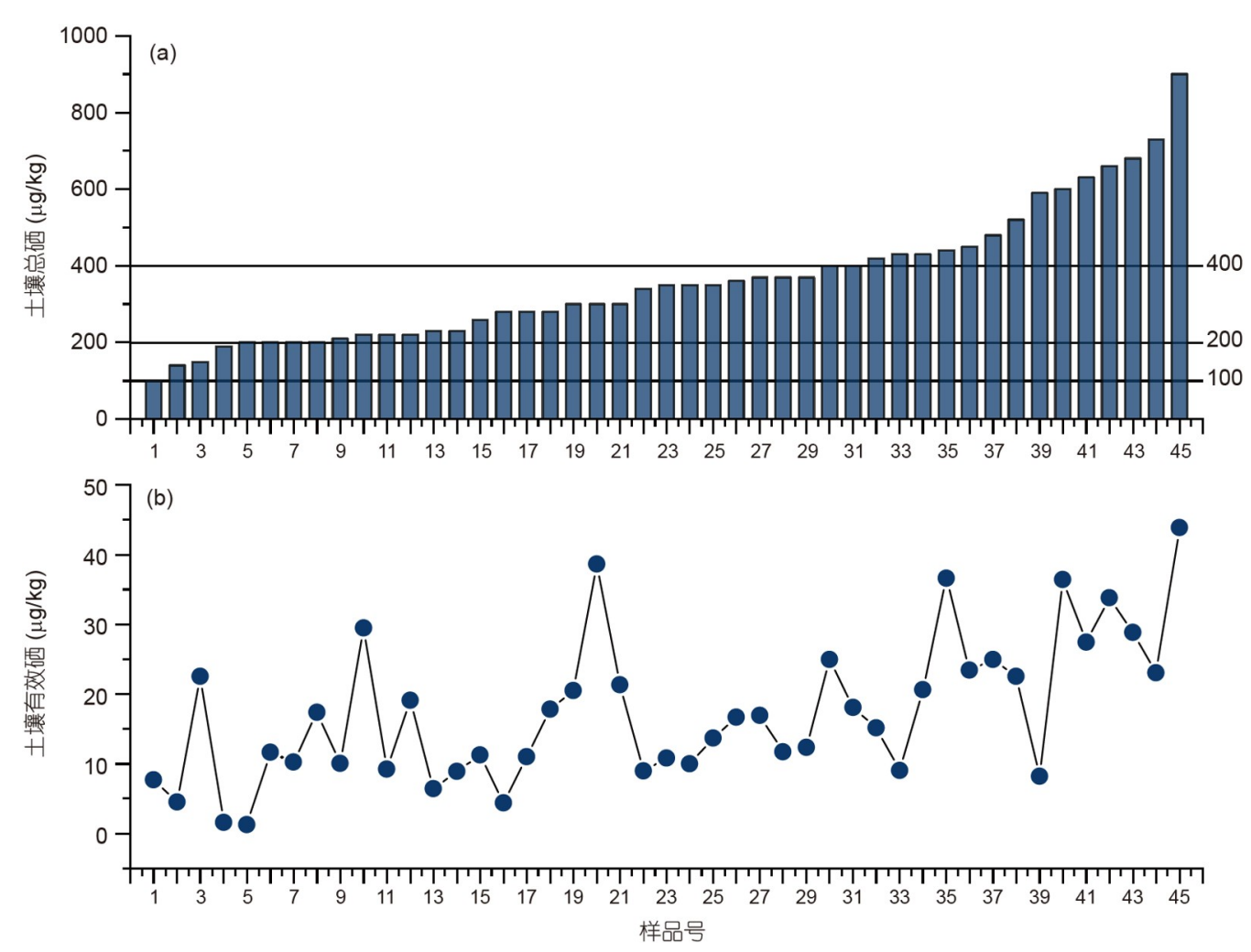

图 2 (网络版彩色)土壤总硒和有效硒含量. 总硒含量 $100 、 200$ 及 $400 \mu \mathrm{g} / \mathrm{kg}$ 为土壤分类界限

Figure 2 (Color online) Soil total and bioavailable selenium contents. 100, 200 and $400 \mu \mathrm{g} / \mathrm{kg}$ were classification limits of soil with selenium

\section{2 土壤线虫群落结构特征}

对线虫群落进行NMDS分析, 克鲁斯卡尔(Kruskal) 应力值为 0.18 , 达到较理想的拟合水平. 不同颜色的点 代表不同硒含量样本, 点之间的距离表示差异程度(图 3). 结果表明, 不同硒含量土壤中, 线虫群落结构区分 明显, 尤其是低硒土壤和高硒土壤, 样本组间距离较大, 说明在硒含量影响下的线虫群落结构差异显著.

在所有土壤样品中共发现 31 属线虫(图4). 食细菌 线虫和植食性线虫种类比较丰富, 被检测到的均有 11 属; 食真菌线虫和杂-捕食线虫种类较少, 检测到的分 别只有5和4属. 在低硒组内, 共有线虫 30 属; 中硒组内, 共有线虫27属; 高硒组内, 共有线虫24属. 样品内的优 势线虫属是潜根属(Hirschmanniella), 在低硒、中硒和 高硒组中相对丰度分别为 $42.3 \% 、 52.7 \%$ 和 $51.2 \%$. 拟 丽突属(Acrobeloides)和丝尾垫刃属(Filenchus)在低硒 组相对丰度较高, 分别为 $11.0 \%$ 和 $10.4 \%$, 在高硒组相对 丰度减小到 $3.3 \%$ 和 $2.2 \%$. 原杆属(Protorhabditis)、中杆 属(Mesorhabditis)、真滑刃属(Aphelenchus)、艾普鲁斯 属(Aprutides)和鳌属(Pungentus)在低硒及中硒组内相
对丰度均较低, 在高硒组中则未检出.

不同硒含量组的线虫类群相对丰度见下图5. 不同 $c-p$ 值的线虫类群中, $c p-3$ 为优势类群, 其相对丰度在不 同硒含量组间达到 $64.0 \% \sim 76.2 \%$, 其次为 $c p-2$ 类群, 相 对丰度为 $20.7 \% \sim 35.7 \%$. $c p-1$ 和 $c p-4$ 都为稀有类群, 线 虫数量较少, 本研究中未检出 $c p-5$ 的线虫. $c p-1 、 c p-3$ 及 $c p-4$ 线虫相对丰度在不同硒含量组间无显著差异, 而低硒组 $c p-2$ 线虫相对丰度显著高于中硒组和高硒组 $(P<0.05)$.

各组内植食性线虫 $\mathrm{PP}$ 为优势类群, 相对丰度为 $67.6 \% \sim 80.8 \%$, 随后相对丰度较大的依次为食细菌线虫 $\mathrm{Ba} 、$ 食真菌线虫 $\mathrm{Fu}$, 杂-捕食线虫数量OP较少, 低硒组 没有观察到该类群. 食细菌线虫、植食性线虫及杂-捕 食线虫相对丰度在各组间无显著差异, 高硒组中食真 菌线虫 $\mathrm{Fu}$ 相对丰度显著低于低硒组 $(P<0.05)$.

\section{3 土壤线虫群落生态指数}

不同硒含量土壤线虫密度及群落生态指数如表 1 所示. 硒含量对土壤线虫密度、F/B值、及结构指数SI 有显著影响. 低硒组线虫密度最小, 仅为 $249.37 \mathrm{ind} /$ 


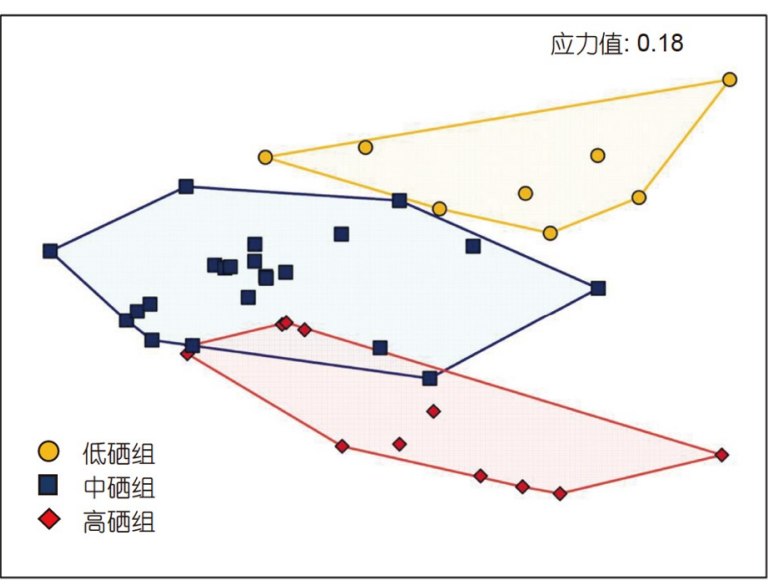

图 3 (网络版彩色)不同硒含量土壤线虫群落的NMDS排序图

Figure 3 (Color online) NMDS ordination of nematode community in soil with different selenium levels

$100 \mathrm{~g}$ 干土，中硒组和高硒组线虫密度显著高于低硒组， 分别为 470.57 和 $543.66 \mathrm{ind} / 100 \mathrm{~g}$ 干土. 低硒组 $F / B$ 值为 0.52 , 显著高于中硒组和高硒组. 低硒组、中硒组及高 硒组土壤线虫SI分别为 $69.83 、 48.56$ 及 43.40 , 低硒组SI
显著高于中硒组和高硒组. 其他线虫群落生态指数在 不同硒含量组土壤间无显著差异.

\section{4 相关性分析}

对环境因子(总硒、有效硒、 $\mathrm{pH}$ 和含水量)及线虫 指标(密度、属丰度、分类群丰度及生态指数)进行相 关性分析，篮选作图如下(图6). 拟丽突属线虫相对丰度 和土壤总硒呈显著负相关关系，相关系数 $r$ 值为 -0.335 $P<0.05)$; 连胃属 $($ Chronogaster $)$ 线虫相对丰度与土壤总 硒及有效硒含量均呈显著正相关关系， $r$ 值分别为 0.446 $(P<0.01)$ 和0.339 $(P<0.05)$; 同样地, 棱咽属(Prismatolai$n u s)$ 线虫相对丰度与土壤总硒及有效硒含量也呈显著正 相关关系， $r$ 值分别为 $0.375(P<0.05)$ 和 $0.312(P<0.05)$; 原杆属、中杆属、头叶属 (Cephalobus)、拟绕线属 (Anaplectus)、绕线属(Plectus)、艾普鲁斯属和裸矛属 (Psilenchus)显著受 $\mathrm{pH}$ 和土壤水分调控 $(P<0.05)$.

线虫密度和土壤总硒土壤有效硒均呈显著正相关 关系，相关系数 $r$ 值分别为 $0.536(P<0.01)$ 和 $0.475(P<$

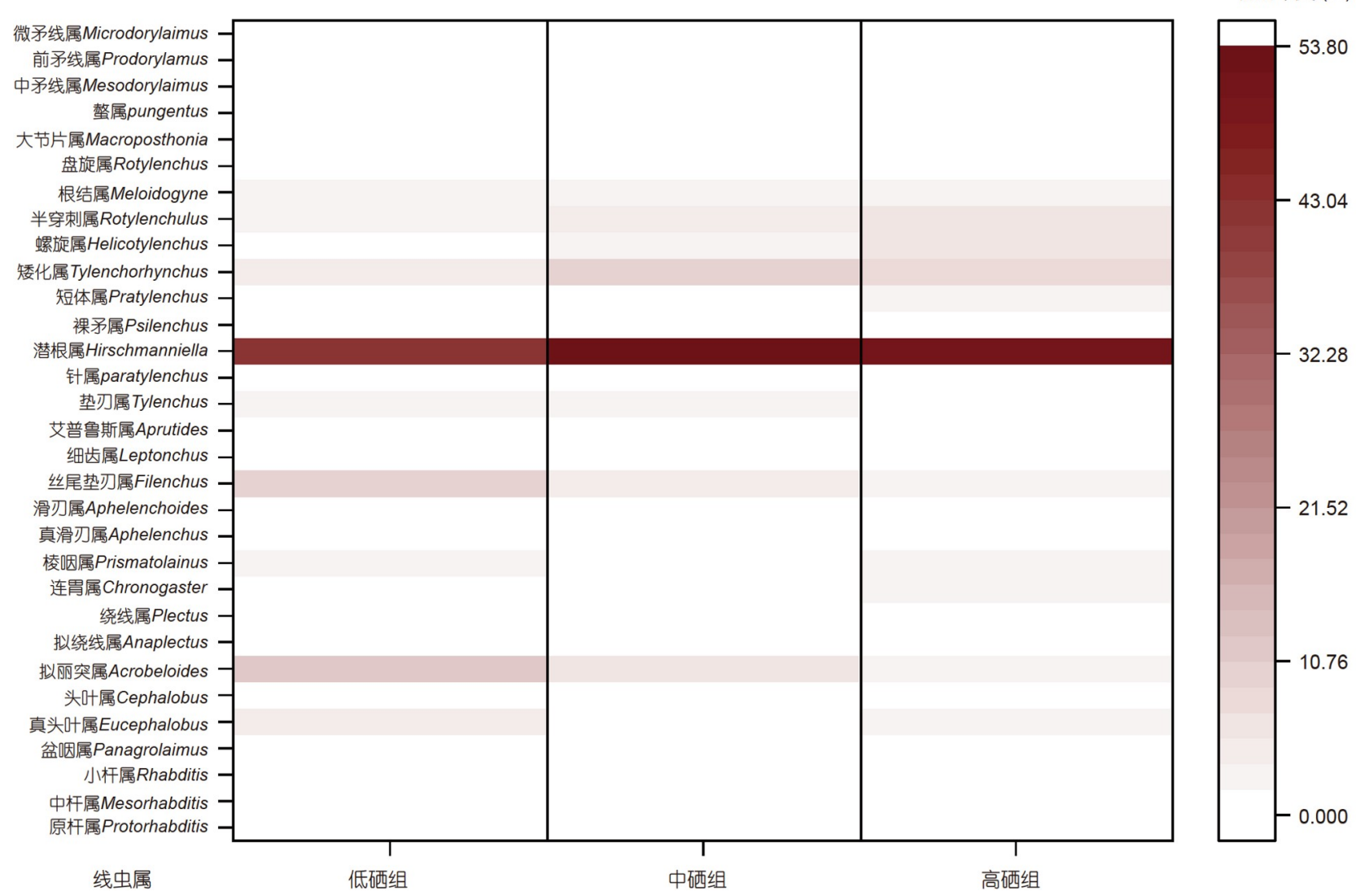

图 4 (网络版彩色)土壤线虫群落属相对丰度图

Figure 4 (Color online) Relative abundance of nematode genus in soil 


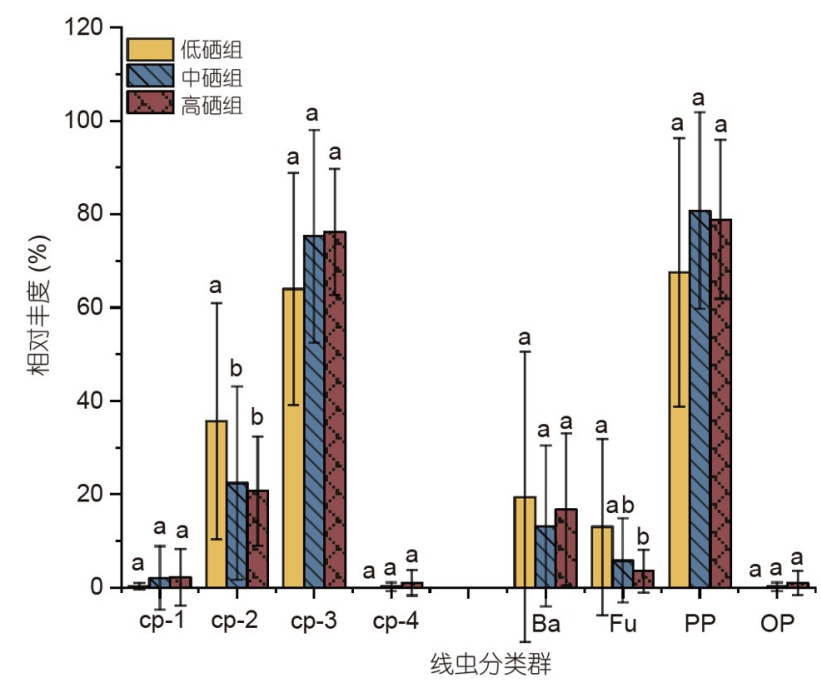

图 5 (网络版彩色)土壤线虫分类群相对丰度图. 字母a、b不同表示 组间存在显著差异 $(P<0.05)$

Figure 5 (Color online) Relative abundance of nematode taxa in soil. Letters $\mathrm{a}$ and $\mathrm{b}$ represent significant differences on soil with different selenium levels at 0.05 level

$0.01)$; 食真菌线虫 $\mathrm{Fu}$ 相对丰度及 $\mathrm{F} / \mathrm{B}$ 值和土壤有效硒存 在显著负相关性, $r$ 值分别为 $-0.307(P<0.05)$ 和 -0.338 $(P<0.05)$; 结构指数SI和土壤总硒呈负相关关系, $r$ 值 为 $-0.521(P<0.01)$.

\section{3 讨论}

我国硒资源总量丰富, 但分布极不均匀, 如东北平 原是典型缺硒区, 土壤总硒含量为 $10 \sim 5300 \mu \mathrm{g} / \mathrm{kg}$, 平均 含量为 $184 \mu \mathrm{g} / \mathrm{kg}^{[27]}$. 而在湖北恩施的一些硒中毒地区, 土壤总硒含量可达 $594380 \mu \mathrm{g} / \mathrm{kg}^{[28]}$. 本研究区域桂平 市, 土壤硒含量适中, 属于天然富硒区. 由于水田土壤 比旱地土壤不易富集硒元素 ${ }^{[29]}$, 除了高硒样点, 本研究 还存在一些低硒和中硒样点.

随总硒和有效硒含量增加，土壤线虫总丰度显著 增大，表明在本研究的硒浓度范围内(100 900 $\mu \mathrm{g} / \mathrm{kg})$, 土壤硒可以促进土壤线虫的繁殖生长. Weiss和Lar- $\mathrm{ink}^{[30]}$ 对农田土壤施用添加了锌的污泥后，农田土壤锌 含量从 $198 \mathrm{mg} / \mathrm{kg}$ 增加到 $360 \mathrm{mg} / \mathrm{kg}$, 土壤线虫总丰度也 从 $4481 \mathrm{ind} / 100 \mathrm{~g}$ 增加到7216 ind/100 g干土. Georgieva 等人 ${ }^{[15]}$ 和Bakonyi等人 ${ }^{[18]}$ 也有类似的发现, 这可能是由 于锌的施用刺激植物生长和微生物量, 从而间接地有 利于植食性线虫和食微线虫的繁殖. 在本研究中, 硒含 量与植食性线虫及食细菌线虫丰度无显著相关性, 但 是从图4可以看出, 相对于低硒组, 高硒组植食性潜根 属线虫丰度相对增大. 潜根属线虫适应厌氧条件, 这使 得它成为水稻-土壤淹水系统内最丰富的线虫物种 ${ }^{[31]}$, 作为优势属其相对丰度的变化很大程度地影响着整个 群落丰度. 硒能促进植物生长, 保护植物免受病原体侵 害 ${ }^{[32]}$, 间接地有利于植食性的潜根属线虫生长, 最终使得 线虫总丰度增加. 需要注意的是, 潜根属线虫寄生于水稻 根部, 大量生长会对作物产生危害, 造成产量损失 ${ }^{[33]}$.

土壤线虫属是构成线虫群落的单位，当土壤生态 系统健康受到干扰, 线虫个体首先作出响应, 个体的数 量减少会引起线虫属丰度的变化. 因此, 各种线虫属的 相对/绝对丰度可作为生物指标来监测土壤受干扰程 度. 在受重金属污染 $(\mathrm{Cu} 、 \mathrm{~Pb} 、 \mathrm{Zn})$ 的稻田土壤中, 相比 对照组, 托布利属(Tobrilus)和拟鞘属 (Hemicrionemoides) 相对丰度显著减小 ${ }^{[34]}$, 这两种线虫可作为重金 属污染的敏感指示生物. 拟丽突属线虫是一种 $c-p$ 值为 2 的食细菌线虫, 广泛分布于各种土壤中. 研究表明, 重 金属暴露可显著抑制拟丽突属线虫的生长繁殖及产卵 数, 且抑制程度和重金属浓度相关 ${ }^{[35]}$. Lü等人 ${ }^{[25]}$ 的研究 也指出高浓度重金属污染土壤中拟丽突属的丰度低于 未污染土壤. 本研究中, 相比于低硒组和中硒组, 高硒 组土壤中拟丽突属线虫相对丰度减小, 拟丽突属线虫 相对丰度和总硒呈显著负相关关系. 说明拟丽突属可 能是对硒敏感的线虫属，硒在较高浓度时会抑制土壤 内的硒敏感线虫, 造成其相对丰度减小. 拟丽突属线虫 和 $\mathrm{pH}$ 及土壤含水量不存在显著相关性，因此可作为不 同环境条件下水稻土壤硒水平的生物指示者. 相对低

\section{表 1 不同硒含量土壤线虫群落生态指数a)}

Table 1 Nematode ecological indexes in soil with different selenium levels

\begin{tabular}{ccccccccccc}
\hline & TN & F/B & $H^{\prime}$ & $S$ & TD & $J^{\prime}$ & MI & SI & EI & NCR \\
\hline 低硒组 & $249.37^{\mathrm{b}}$ & $0.52^{\mathrm{a}}$ & $0.80^{\mathrm{a}}$ & $15.63 \mathrm{a}$ & $1.63^{\mathrm{a}}$ & $0.63^{\mathrm{a}}$ & $2.64^{\mathrm{a}}$ & $69.83^{\mathrm{a}}$ & $85.60^{\mathrm{a}}$ & $0.66^{\mathrm{a}}$ \\
中硒组 & $470.57^{\mathrm{a}}$ & $0.34^{\mathrm{b}}$ & $1.14^{\mathrm{a}}$ & $12.48^{\mathrm{a}}$ & $1.64^{\mathrm{a}}$ & $0.66^{\mathrm{a}}$ & $2.74^{\mathrm{a}}$ & $48.56^{\mathrm{b}}$ & $89.12^{\mathrm{a}}$ & $0.75^{\mathrm{a}}$ \\
高硒组 & $543.66^{\mathrm{a}}$ & $0.32^{\mathrm{b}}$ & $1.29^{\mathrm{a}}$ & $11.07^{\mathrm{a}}$ & $1.79^{\mathrm{a}}$ & $0.61^{\mathrm{a}}$ & $2.59^{\mathrm{a}}$ & $43.40^{\mathrm{b}}$ & $86.17^{\mathrm{a}}$ & $0.76^{\mathrm{a}}$ \\
\hline
\end{tabular}

a) $\mathrm{TN}$ 为线虫密度(ind/ $100 \mathrm{~g}$ 干土), 字母 $\mathrm{a} 、 \mathrm{~b}$ 不同表示组间存在显著差异 $(P<0.05)$ 


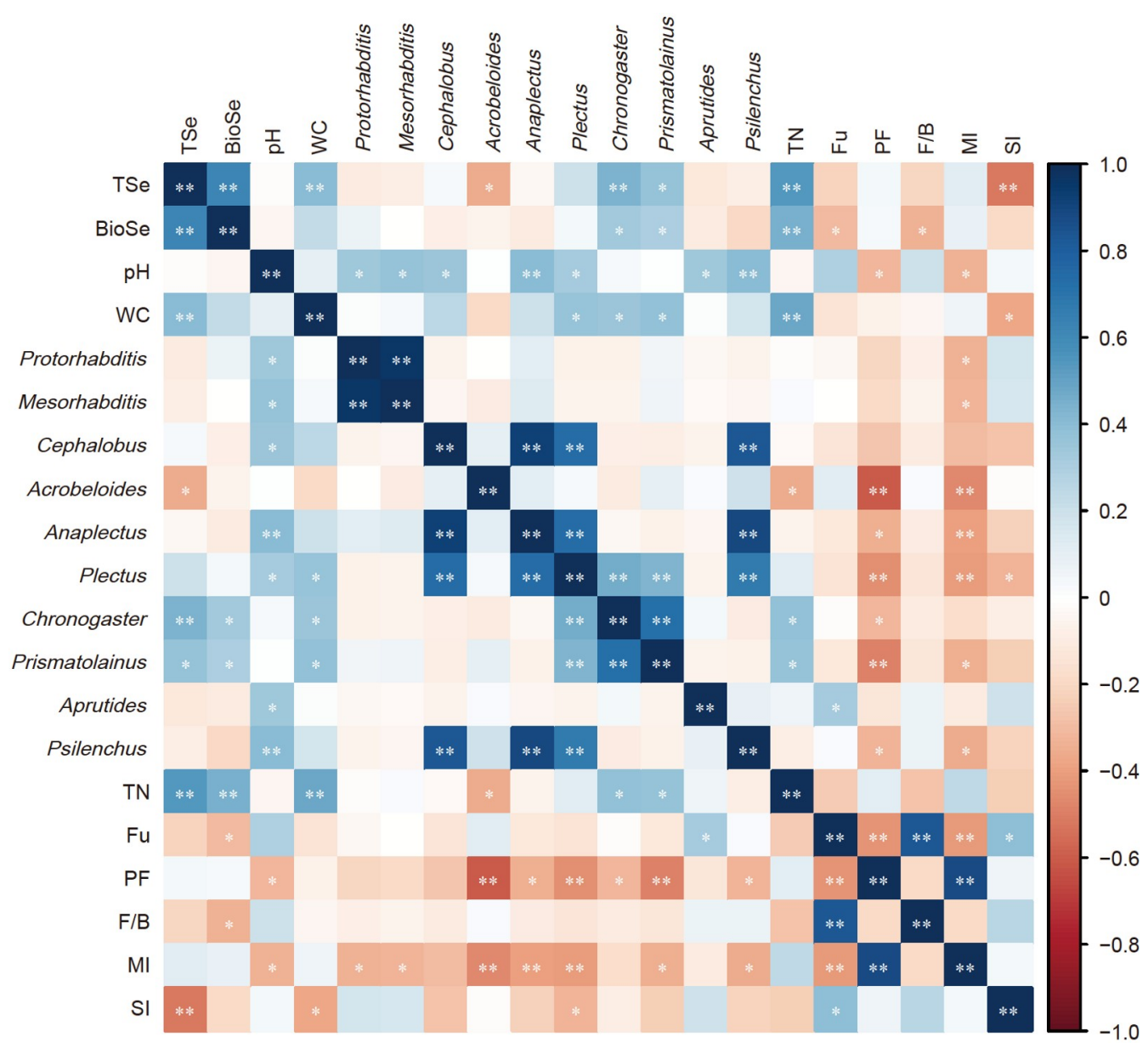

图 6 环境因子和线虫群落指数相关性热图. TSe为土壤总硒, BioSe为土壤有效硒, WC为土壤含水量. *表示显著相关 $(P<0.05)$, **表示极显著 相关 $(P<0.01)$

Figure 6 Correlation between environmental factors and nematode indexes. TSe is soil total selenium content, BioSe is soil bioavailable selenium content, and WC is soil water content. * represents significant correlation at 0.05 level, and ** represents significant correlation at 0.01 level

硒组, 高硒组丝尾垫刃属线虫相对丰度减小, 但是该线 虫属在各种类型土壤中所占比例较小 ${ }^{[36]}$, 尚未有其他 研究表明其对硒或重金属胁迫敏感. 连胃属和棱咽属 线虫相对丰度和总硒呈显著正相关性，说明其对硒耐 受性较高, 在适宜区间内, 硒能作为有益元素促进该线 虫生长繁殖.

土壤线虫在 4 个营养类群中的分布反映了它们的 食物网关系，在不同 $c-p$ 值间的分布反映了线虫采取的 生活史策略. 本研究中, 不同硒含量土壤中, 线虫类群 分布存在显著差异. 其中 $c p-2$ 的线虫及食真菌线虫Fu对 硒较为敏感. 相对于低硒土壤, $c p-2$ 线虫在高硒土壤中 相对丰度显著降低. $c p-2$ 线虫相对丰度降低可能是由于 硒敏感拟丽突属和丝尾垫刀属线虫相对丰度下降造成 的, 这两种线虫的 $c-p$ 值均为 2 . 相对于低硒组, 高硒组土
壤食真菌线虫 $\mathrm{Fu}$ 相对丰度显著下降，且 $\mathrm{Fu}$ 和土壤总硒 呈显著负相关性. 食真菌线虫通过捕食真菌这一食物 链参与土壤物质和能量流动 ${ }^{[37,38]}$, 食真菌线虫 $\mathrm{Fu}$ 相对 丰度降低，可能会抑制真菌主导的复杂有机质分解过 程, 减慢土壤养分循环. 食真菌线虫对硒的响应也可能 是通过与真菌的相互作用实现的. 硒对部分真菌具有 抑制作用, 可帮助植物抵抗多种真菌病原体 ${ }^{[39,40]}$. 食真 菌线虫以真菌为食，研究显示在培养基上其数量与真 菌数量具有正相关性 ${ }^{[41]}$. 土壤有效硒是总硒中具有生 物有效性的部分, 因此, 土壤中有效硒浓度增加可能会 影响土壤真菌数量，从而间接导致食真菌线虫 $\mathrm{Fu}$ 相对 丰度及F/B值的减小.

除分类群相对丰度外，线虫的多种生态指数也可 有效反映出土壤线虫群落的多样性和结构差异. 牵牛 
花盆栽试验显示, 实验组土壤施加镉 $(5 \mathrm{mg} / \mathrm{kg})$ 和录 $(20 \mathrm{mg} / \mathrm{kg}) 3$ 个月后, 相比于对照组, 土壤线虫的多样性 指数 $H^{\prime}$ 、均匀度 $J^{\prime}$ 及营养多样性指数TD均显著减小 ${ }^{[25]}$. 而本研究中, 不同硒含量土壤线虫属数 $S$ 、多样性指数 $H^{\prime}$ 、均匀度 $J^{\prime}$ 及营养多样性指数 TD均无显著差异. 这 可能是由于土壤硒含量总体上较低, 且硒的生物毒性 要远小于重金属镉和永, 所以土壤中硒含量变化未对这 些生态指数产生显著影响. 结构指数SI指示土壤食物网 复杂程度 ${ }^{[23]}$. 在本研究中, 线虫的群落结构指数SI和土 壤总硒呈显著负相关, 土壤硒含量越高, 结构指数SI越 低. 这可能是由于一部分对硒敏感的土壤动物死亡, 进 而缩短食物链, 降低食物网复杂程度. 与我们的结果类 似, $\mathrm{Li}^{\text {等人 }}{ }^{[42]}$ 的研究表明, 受重金属污染的土壤中杂食捕食性线虫 $\mathrm{OP}$ 的减少或消失降低了土壤食物网的复杂 性, 导致结构指数SI值降低. 结构指数减小说明土壤食 物网受到扰动, 生物多样性降低, 土壤生态系统稳定性 减小, 指示硒含量变化对土壤生态系统已经造成影响. 因此, 在一定范围内, 可以利用结构指数SI作为生物指 标指示土壤硒是否对土壤生态系统产生干扰及干扰程 度. 通道指数NCR指示土壤内有机质分解途径. 硒和通 道指数无显著相关性, 大部分采样点土壤通道指数 NCR 大于 0.5 , 指示土壤中主要分解者是细菌. 一般来说, 水稻 土壤水分高, 细菌数量多于真菌, 所以在水稻土壤中, 有 机质分解以细菌分解为主. 如刘满强等人 ${ }^{[43]}$ 计算线虫通 道指数NCR结果也表明水稻土壤中主要分解者是细菌.
富集指数EI可以反映土壤食物网资源的可利用性和初 级分解者对资源的响应 ${ }^{[4]}$, 硒对其无显著影响. 成熟度 指数MI相当于整个群落的加权 $c-p$ 值 ${ }^{[23]}$, 本研究中所有 样点内 $c-p$ 值为 2 和 3 的线虫占据主要地位, 成熟度指数 都在2 3之间, 硒和该指数也无显著相关性.

在农田生态系统中, 大量研究指出, 土壤质地、 $\mathrm{pH}$ 、含水量及氮磷钾等会影响线虫群落 ${ }^{[45,46]}$. 本研究 中, 土壤 $\mathrm{pH}$ 及含水量和多个线虫指标具有显著相关性, 也证实了土壤理化性质对线虫的影响作用. 在未来的 研究中, 为了拓展和深化本研究内容, 可以建立盆栽试 验或大田试验, 确认土壤硒元素对线虫群落的作用及 线虫的指示作用, 还可以寻找更高硒含量天然土壤, 结 合土壤理化性质对土壤线虫群落进行分析.

\section{4 结论}

在总硒含量 100 900 $\mu \mathrm{g} / \mathrm{kg}$ 范围内的天然水稻土壤 中, 土壤硒含量的增加促进了线虫密度、连胃属及棱 咽属相对丰度的增加, 但显著降低拟丽突属相对丰 度、食真菌线虫 $\mathrm{Fu}$ 相对丰度、 $\mathrm{F} / \mathrm{B}$ 值和结构指数 SI值. 相比于低硒组, 高硒组水稻土壤线虫密度增大, 但是土 壤食物网结构简化, 稳定性下降. 这表明, 线虫对硒元 素有敏感响应, 线虫群落对含硒水稻土壤生态系统的 变化表现出一定的指示潜力, 拟丽突属线虫有可能作 为天然富硒水稻土硒水平的监测指标, 对富硒水稻土 壤进行评价、管理及修复.

\section{参考文献}

1 Fairweather-Tait S J, Bao Y, Broadley M R, et al. Selenium in human health and disease. Antioxid Redox Signal, 2011, 14: 1337-1383

2 Combs G F. Selenium in global food systems. Br J Nutr, 2001, 85: 517-547

3 National Health and Family Planning Commission of the People's Republic of China. WS/T 578.3-2017 Chinese Dietary Reference Intakes-Part 3: Trace Element (in Chinese). 2017 [中华人民共和国卫生和计划生育委员会. WS/T 578.3-2017中国居民膳食营养素参考摄人量第3部分. 2017]

4 Duan M L, Fu D D, Wang S S, et al. Effects of different selenite concentrations on plant growth, absorption and transportation of selenium in four different vegetables (in Chinese). Acta Sci Circumst, 2011, 31: 658-665 [段曼莉, 付冬冬, 王松山, 等. 亚硒酸盐对四种蔬菜生长、吸收及转运 硒的影响. 环境科学学报, 2011, 31: 658-665]

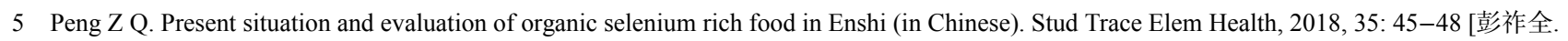
恩施州富有机硒食品现状及评价. 微量元素与健康研究, 2018, 35: 45-48]

6 Manojlović M S, Lončarić Z, Cabilovski R R, et al. Biofortification of wheat cultivars with selenium. Acta Agric Scand, 2019, 69: 715-724

7 Oliveira V C, Faquin V, Guimarães K C, et al. Agronomic biofortification of carrot with selenium. Ciênc Agrotec, 2018, 42: 138-147

8 Premarathna L, McLaughlin M J, Kirby J K, et al. Selenate-enriched urea granules are a highly effective fertilizer for selenium biofortification of paddy rice grain. J Agric Food Chem, 2012, 60: 6037-6044

9 Wang Y D, Wang X, Wong Y S. Generation of selenium-enriched rice with enhanced grain yield, selenium content and bioavailability through fertilisation with selenite. Food Chem, 2013, 141: 2385-2393

10 Paz-Ferreiro J, Fu S. Biological indices for soil quality evaluation: Perspectives and limitations. Land Degrad Dev, 2016, 27: 14-25 
11 Neher D A. Role of nematodes in soil health and their use as indicators. J Nematol, 2001, 33: 161-168

12 Ekschmitt K, Bakonyi G, Bongers M, et al. Nematode community structure as indicator of soil functioning in European grassland soils. Eur J Soil Biol, 2001, 37: 263-268

13 Bongers $\mathrm{T}$, Ilieva-Makulec $\mathrm{K}$, Ekschmitt $\mathrm{K}$. Acute sensitivity of nematode taxa to $\mathrm{CuSO}_{4}$ and relationships with feeding-type and life-history classification. Environ Toxicol Chem, 2010, 20: 1511-1516

14 Shao Y, Zhang W, Shen J, et al. Nematodes as indicators of soil recovery in tailings of a lead/zinc mine. Soil Biol Biochem, 2008, 40: 2040-2046

15 Georgieva S S, McGrath S P, Hooper D J, et al. Nematode communities under stress: The long-term effects of heavy metals in soil treated with sewage sludge. Appl Soil Ecol, 2002, 20: 27-42

16 Park B Y, Lee J K, Ro H M, et al. Short-term effects of low-level heavy metal contamination on soil health analyzed by nematode community structure. Plant Pathol J, 2016, 32: 329-339

17 Pen-Mouratov S, Shukurov N, Steinberger Y. Influence of industrial heavy metal pollution on soil free-living nematode population. Environ Pollut, 2008, 152: 172-183

18 Bakonyi G, Nagy P, Kádár I. Long-term effects of heavy metals and microelements on nematode assemblage. Toxicol Lett, 2003, 140-141: 391401

19 Long Z, Yuan L, Hou Y, et al. Spatial variations in soil selenium and residential dietary selenium intake in a selenium-rich county, Shitai, Anhui, China. J Trace Elem Med Biol, 2018, 50: 111-116

20 Zhao C, Ren J, Xue C, et al. Study on the relationship between soil selenium and plant selenium uptake. Plant Soil, 2005, 277: 197-206

21 Zhang W, Liu M Q, He Y Q, et al. Responses of soil nematode communities to long-term application of inorganic fertilizers in upland red soil (in Chinese). Chin J Appl Ecol, 2014, 25: 2361-2368 [张微, 刘满强, 何园球, 等. 长期施用不同无机肥对旱地红壤线虫群落的影响. 应用生态学 报, 2014, 25: 2361-2368]

22 Wang M W, Liu Y D, Cheng X Y, et al. Response of soil nematode community to cultivation in upland red soil relative to cultivation history and its significance as indicator (in Chinese). Acta Pedol Sin, 2016, 53: 510-522 [王明伟, 刘雨迪, 陈小云, 等. 旱地红壤线虫群落对不同耕作年限的 响应及指示意义. 土壤学报, 2016, 53: 510-522]

23 Ferris H, Bongers T, de Goede R G M. A framework for soil food web diagnostics: Extension of the nematode faunal analysis concept. Appl Soil Ecol, 2001, 18: 13-29

24 Bongers T, Bongers M. Functional diversity of nematodes. Appl Soil Ecol, 1998, 10: 239-251

25 Lü Y, Chen X, Xue W F, et al. Short-term effects of cadmium and mercury on soil nematode communities in a pot experiment. Helminthologia, 2020, 57: 145-153

26 Li J X, Zhang G D. Geochemical Environmental Characteristics of Selenium Deficiency and Excess in Human Body and its Prediction (in Chinese). Beijing: Geological Publishing House, 2000 [李家熙, 张光弟. 人体硒缺乏与过剩的地球化学环境特征及其预测. 北京: 地质出版社, 2000]

27 Dai H M, Gong C D, Dong B, et al. Distribution of soil selenium in the northeast china plain and its influencing factors (in Chinese). Acta Pedol Sin, 2015, 52: 1356-1364 [戴慧敏, 宫传东, 董北, 等. 东北平原土壤硒分布特征及影响因素. 土壤学报, 2015, 52: 1356-1364]

28 Zhu J M, Zuo W, Qing H B, et al. An investigation on the source of soil Se in Yutangba, Enshi: Evidance from native selenium (in Chinese). Acta Miner Sin, 2008, 28: 397-400 [朱建明, 左维, 秦海波, 等. 恩施硒中毒区土壤高硒的成因: 自然硒的证据. 矿物学报, 2008, 28: 397-400]

29 Huang C L, Song M Y, Wei Y C. Study on selenium contents of typical selenium-rich soil in the middle area of Zhejiang and its influencing factors (in Chinese). Environ Sci, 2013, 34: 269-274 [黄春雷, 宋明义, 魏迎春. 浙中典型富硒土壤区土壤硒含量的影响因素探讨. 环境科学, 2013, 34: 269-274]

30 Weiss B, Larink O. Influence of sewage sludge and heavy metals on nematodes in an arable soil. Biol Fert Soils, 1991, 12: 5-9

31 Liao J L, Feng Z X, Li S M, et al. Species of Hirschmanniella on rice and their distribution in China. Nematol Mediterr, 2000, 28: 107-110

32 EI Mehdawi A F, Pilon-Smits E A H. Ecological aspects of plant selenium hyperaccumulation. Plant Biol, 2011, 14: 1-10

33 Xie Z C. 1dentification of nematodes in rice root and pathogenicity of Hirschmanniella and Meloidogyne to rice (in Chinese). Doctor Dissertation. Fuzhou: Fujian Agriculture and Forestry University, 2007 [谢志成. 水稻根部线虫鉴定及潜根线虫根结线虫对水稻的致病性. 博士学位论文. 福州: 福建农林大学, 2007]

34 Wang Y L, Wang H H, Liao J L, et al. Effects of heavy metal contamination on nematode communities in paddy soils of an e-waste recycling area (in Chinese). J Agro-Environ Sci, 2015, 34: 874-881 [王赢利, 王宏洪, 廖金铃, 等. 电子垃圾拆解地重金属污染对稻田土壤线虫群落结构的影 响. 农业环境科学学报, 2015, 34: 874-881]

35 Hao W, Jiang Y, Li Q, et al. Subacute toxic effects of lead and cadmium on the nematode Acrobeloides nanus (in Chinese). Asian J Ecotox, 2008, 3 : 139-143 [郝伟, 姜勇, 李琪, 等. 重金属铅, 镉对矮小拟丽突线虫(Acrobeloides nanus)的亚急性毒性作用. 生态毒理学报, 2008, 3: 139-143]

36 Salamún P, Reno M, Kucanová E, et al. Nematodes as bioindicators of soil degradation due to heavy metals. Ecotoxicology, 2012, 21: 2319-2330

37 Xiao H F. Infiuences of bacterial-feeding nematodes on bacterial numbers, community structure studied in soil (in Chinese). Doctor Dissertation. 
Nanjing: Nanjing Agricultural University, 2010 [肖海峰. 土壤食细菌线虫对微生物数量和群落结构的影响. 博士学位论文. 南京: 南京农业大 学, 2010]

38 Wu J H, Song C Y, Chen J K, et al. Effect of microbivorous nematodes on plant growth and soil nutrient cycling: A review (in Chinese). Biodivers Sci, 2007, 15: 124-133 [吴纪华, 宋慈玉, 陈家宽. 食微线虫对植物生长及土壤养分循环的影响. 生物多样性, 2007, 15: 124-133]

39 Cheng Q, Hu C X, Jia W, et al. Selenium reduces the pathogenicity of Sclerotinia sclerotiorum by inhibiting sclerotial formation and germination. Ecotox Environ Safe, 2019, 183: 1-9

40 Hanson B, Garifullina G F, Lindblom S D, et al. Selenium accumulation protects Brassica juncea from invertebrate herbivory and fungal infection. New Phytol, 2010, 159: 461-469

$41 \mathrm{Li} \mathrm{H} \mathrm{X,} \mathrm{Mao} \mathrm{X} \mathrm{F,} \mathrm{Hu} \mathrm{F,} \mathrm{et} \mathrm{al.} \mathrm{Interactions} \mathrm{between} \mathrm{fungal-feeding} \mathrm{nematodes} \mathrm{and} \mathrm{fungi} \mathrm{and} \mathrm{their} \mathrm{effects} \mathrm{on} \mathrm{soil} \mathrm{nitrogen} \mathrm{mineralization} \mathrm{(in}$ Chinese). Chin J Appl Ecol, 2004, 15: 2304-2308 [李辉信, 毛小芳, 胡锋, 等. 食真菌线虫与真菌的相互作用及其对土壤氮素矿化的影响. 应 用生态学报, 2004, 15: 2304-2308]

42 Li Q, Zhong S, Li F, et al. Nematode community structure as bioindicator of soil heavy metal pollution along an urban rural gradient in southern Shenyang, China. Int J Environ Pollut, 2011, 45: 297-309

43 Liu M Q, Huang J H, Chen X Y, et al. Aboveground herbivory by the brown planthopper (Nilaparvata lugens) affects soil nematode communities under difkrent rice varieties (in Chinese). Biodivers Sci, 2009, 17: 431-439 [刘满强, 黄菁华, 陈小云, 等. 地上部植食者褐飞禹对不同水稻品种 土壤线虫群落的影响. 生物多样性, 2009, 17: 431-439]

44 Song M, Li X, Jing S, et al. Responses of soil nematodes to water and nitrogen additions in an old-field grassland. Appl Soil Ecol, 2016, 102: 53-60

45 Jiao J G, Liu B B, Mao M, et al. Characteristics of soil nematode community of different agricultural areas in Jiangsu Province, China (in Chinese). Chin J Appl Ecol, 2015, 26: 3489-3496 [焦加国, 刘贝贝, 毛妙, 等. 江苏省不同农业区土壤线虫群落分布特征. 应用生态学报, 2015, 26: 3489-3496]

46 Sun Z, Liu M Q, Gui J, et al. Reduction of nitrogen amendment and irrigation influences paddy soil nematode community (in Chinese). Chin J Ecol, 2014, 33: 659-665 [孙震, 刘满强, 桂娟, 等. 减施氮肥和控制灌溉对稻田土壤线虫群落的影响. 生态学杂志, 2014, 33: 659-665] 


\section{Nematode structure and its indication in natural selenium-rich paddy soil of Guiping City, Guangxi Zhuang Autonomous Region}

Jiaping Song', Linxi Yuan ${ }^{2}$, Xiaodong Liu ${ }^{1}$, Yongxian Liu ${ }^{3}$, Zhangmin Wang ${ }^{4}$, Qingqing Chen ${ }^{4}$, Zezhou Zhang ${ }^{4}$, Zedong Long ${ }^{5}$, Jinyu Lin ${ }^{1} \&$ Xuebin Yin ${ }^{1,4 *}$

${ }^{1}$ School of Earth and Space Sciences, University of Science and Technology of China, Hefei 230026, China;

${ }^{2}$ Department of Health and Environmental Sciences, Xi'an Jiaotong-Liverpool University, Suzhou 215123, China;

${ }^{3}$ Institute of Agricultural Resources and Environment, Guangxi Academy of Agricultural Sciences, Nanning 530007, China,

4 Jiangsu Bio-Engineering Research Center for Selenium, Suzhou 215123, China;

${ }^{5}$ Soil and Fertilizer Institute, Hunan Academy of Agricultural Sciences, Changsha 410125, China

* Corresponding author, E-mail: xbyin@ustc.edu.cn

Selenium is one of the essential trace elements for humans. Adequate intake of Se may reduce the body risk of some types of cancer and protect the liver and resist oxidation. Agricultural products are the main source of selenium for the human. Therefore, the development of selenium-rich agriculture is of great significance to improving human health. However, no research on ecological security assessment in selenium-rich soils has been carried out yet. Soil organisms are the primary regulators of the soil material cycle and energy flow process. Soil fauna has been gradually used in soil environmental quality monitoring with widespread attention to the diversity of soil fauna and its ecological importance. Nematode is the most abundant metazoan in soil, and its community structure and diversity indicate energy flow and environmental quality in soil ecosystem. In this study, natural Se-rich paddy soils $(100-900 \mu \mathrm{g} / \mathrm{kg} \mathrm{Se})$ in Guiping City, Guangxi Zhuang Autonomous Region were classified into three groups (low Se group, medium Se group and high Se group) for nematode communities analysis. Non-metric multidimensional scaling ordination of nematode community showed that soil nematode community structure in different groups was significantly different. A total of 31 genera of nematodes were found in all the samples, and the sensitive nematode genera (Acrobeloides, Filenchus, etc.) exhibited different distribution among the different groups. Compared with the low Se group, the density of nematodes in medium Se group and high Se group increased while the relative abundance of $c p-2$ nematodes, the relative abundance of fungivores, the F/B value and structural index SI decreased significantly. Correlation analysis showed that nematode density, the relative abundance of Chronogaster and Prismatolainus increased with the increase of soil Se contents, while the relative abundance of Acrobeloides, the relative abundance of fungivores, F/B value and SI decreased. In conclusion, selenium increased nematode density but simplified soil food web with inhibited sensitive genera. Our study primarily demonstrated that a high soil selenium level might bring a negative effect on nematode community in paddy soil. It is necessary to regulate soil selenium levels to protect soil biodiversity when utilizing natural selenium-rich soil or fertilizers to produce selenium-rich agricultural products. Additionally, considering the sensitive response to selenium, nematode could be used as a bioindicator for natural selenium-rich paddy soil management. This study provides a reference for the scientific evaluation of ecological security of natural selenium-rich paddy soil. For further research in the indication of nematodes on soil selenium, biological and abiotic factors of the soil should be taken into account in this system.

paddy soil, selenium, nematode, structure index, Acrobeloides, bioindicator

doi: 10.1360/TB-2021-0118 